\title{
Single-trial lie detection using a combined fNIRS-polygraph system
}

\author{
M. Raheel Bhutta ${ }^{1}$, Melissa J. Hong ${ }^{2}$, Yun-Hee Kim ${ }^{3}$ and Keum-Shik Hong ${ }^{1,4 *}$ \\ ${ }^{1}$ Department of Cogno-Mechatronics Engineering, Pusan National University, Busan, South Korea, ${ }^{2}$ FIRST 5 Santa Clara \\ County, San Jose, CA, USA, ${ }^{3}$ Department of Physical and Rehabilitation Medicine, Center for Prevention and Rehabilitation, \\ Heart Vascular and Stroke Institute, Samsung Medical Center, Sungkyunkwan University School of Medicine, Samsung \\ Advanced Institute of Health Sciences \& Technology, Sungkyunkwan University, Seoul, South Korea, ${ }^{4}$ School of Mechanical \\ Engineering, Pusan National University, Busan, South Korea
}

\section{OPEN ACCESS}

Edited by:

Nikolai Axmacher,

University of Bochum, Germany

Reviewed by:

Fabian Guénolé,

Centre Hospitalier Universitaire de

Caen, France

Lorena Deuker,

Donders Institute for Brain, Cognition and Behaviour, Netherlands

*Correspondence:

Keum-Shik Hong,

School of Mechanical Engineering,

Pusan National University, 2

Busandaehak-ro, Geumjeong-gu,

Busan 609-735, South Korea

kshong@pusan.ac.kr

Specialty section:

This article was submitted to

Psychoanalysis and

Neuropsychoanalysis,

a section of the journal

Frontiers in Psychology

Received: 11 February 2015

Accepted: 13 May 2015

Published: 02 June 2015

Citation:

Bhutta MR, Hong MJ, Kim Y-H and

Hong K-S (2015) Single-trial lie

detection using a combined

fNIRS-polygraph system.

Front. Psychol. 6:709.

doi: 10.3389/fpsyg.2015.00709
Deception is a human behavior that many people experience in daily life. It involves complex neuronal activities in addition to several physiological changes in the body. A polygraph, which can measure some of the physiological responses from the body, has been widely employed in lie-detection. Many researchers, however, believe that lie detection can become more precise if the neuronal changes that occur in the process of deception can be isolated and measured. In this study, we combine both measures (i.e., physiological and neuronal changes) for enhanced lie-detection. Specifically, to investigate the deception-related hemodynamic response, functional near-infrared spectroscopy (fNIRS) is applied at the prefrontal cortex besides a commercially available polygraph system. A mock crime scenario with a single-trial stimulus is set up as a deception protocol. The acquired data are classified into "true" and "lie" classes based on the fNIRS-based hemoglobin-concentration changes and polygraph-based physiological signal changes. Linear discriminant analysis is utilized as a classifier. The results indicate that the combined fNIRS-polygraph system delivers much higher classification accuracy than that of a singular system. This study demonstrates a plausible solution toward single-trial lie-detection by combining fNIRS and the polygraph.

Keywords: deception, single-trial lie detection, combined fNIRS-polygraph system, hemoglobin change, physiological responses, linear discriminant analysis

\section{Introduction}

Accurate and reliable lie detection represents an intriguing challenge for experts in various scientific fields. The objective of this paper is to enhance the classification accuracy in single trial lie detection paradigm by combining a functional near-infrared spectroscopy (fNIRS) and autonomic data (i.e., electrodermal and respiratory activities). A polygraph, which measures several physiological parameters in the body including respiration, skin conductance, blood pressure, and pulse rate, is widely employed in lie-detection settings (Jung, 1919; Mohamed et al., 2006; Matsuda et al., 2013; Walczyk et al., 2013).

Abbreviations: BCI, Brain-computer interface; DPF, Differential pathlength factor; EDA, Electrodermal activity; EEG, Electroencephalography; ERP, Event-related potentials; fMRI, Functional magnetic resonance imaging; fNIRS, Functional near-infrared spectroscopy; GKT, Guilty knowledge test; HbO, Oxy-hemoglobin; HbR, Deoxy-hemoglobin; LDA, Linear discriminant analysis; LED, Light-emitting diode; NIR, Near-infrared; PAS, Paragon Acquisition System; PFC, Prefrontal cortex; rCBF, Regional cerebral blood flow; SM, Signal mean; SS, Signal slope. 
Many researchers support the hypothesis that direct measurement of brain functions might enable more complete understanding of deception and, therefrom, more accurate and consistent detection of lies. Based on this hypothesis, many researchers have investigated various neurophysiological signals for possible use in lie-detection applications. Among these signals are event-related potentials (ERPs), which are acquired by electroencephalography (EEG) from the scalp (Duncan et al., 2009; Rosenfeld et al., 2012). ERPs are used mostly to test for knowledge about the crime details known only to the criminal involved (Farwell and Donchin, 1991). This type of test is commonly known as a guilty knowledge test (GKT) or concealed information test (CIT) (Zhao et al., 2012; Farahani and Moradi, 2013; Rosenfeld et al., 2013). EEG, with its high temporal resolution, detects brain signals very quickly (Turnip et al., 2011; Cantilena et al., 2012; Soekadar et al., 2014), but the main disadvantage is its poor spatial resolution. The other technique widely used to detect brain areas that are activated in the course of deception is functional magnetic resonance imaging (fMRI). fMRI, with its high spatial resolution relative to that of EEG (Spence et al., 2004; Yuan and Ye, 2013; Liang et al., 2014), can easily localize changes in the regional cerebral blood flow (rCBF). A comprehensive review of fMRI-based deception decoding has found the current fMRI technique to be limited due to high cost of its scanners, bulky size, and its high sensitivity to motion artifacts (Farah et al., 2014).

Researchers therefore have begun to explore another brainimaging technique, fNIRS, which measures brain activity through hemodynamic responses associated with neuron behavior (Kochel et al., 2011; Kamran and Hong, 2013; Santosa et al., 2013; Hong and Nguyen, 2014; Tempest et al., 2014; Naseer and Hong, 2015b), in doing so it provides both topographic (Wolf et al., 2007) and tomographic brain images (Barbour et al., 2001): A topographic image is to depict the brain activities on the brain surface, whereas a tomographic image is to characterize the internal difference in the brain. In the case of ANIRS, the hemodynamic responses in association with the brain activities can be topographically mapped on the brain surface (i.e., intensity map), whereas the dynamics of the vascular responses during the neural activation can be tomographically reconstructed in the $3 \mathrm{D}$ space (Barbour et al., 2001).

Near-infrared (NIR)-spectrum light takes advantage of a $650 \sim 1000 \mathrm{~nm}$ optical window in which skin, tissue, and bone are mostly transparent to NIR light, but wherein oxy-hemoglobin (HbO) and deoxy-hemoglobin (HbR) are strong light absorbers. fNIRS provides a better temporal/spatial resolution trade-off than does EEG or fMRI. A comprehensive comparative evaluation of the respective features of ANIRS and fMRI indicated that fNIRS has a much greater potential for psychiatric and neurological applications, owing specifically to its better portability, simplicity, and insensitivity to motion artifacts (Irani et al., 2007). fNIRS therefore has been used in a great variety of fields, including neuroscience ( $\mathrm{Hu}$ et al., 2011), sports medicine (Quaresima et al., 2003), behavioral studies (Roos et al., 2011; Santosa et al., 2014), clinical medicine (Taillefer and Denault, 2005), pediatrics (Lanfranconi et al., 2014), and brain-computer interface (BCI) (Fazli et al., 2012; Naseer and Hong, 2013, 2015a; Naseer et al., 2014). Recently, small, portable and cost-effective fNIRS systems have extended the application of this modality to outdoor activities such as exercise or those conducted for rehabilitation purposes (Muehlemann et al., 2008; Kim et al., 2011; Bhutta et al., 2014; Khan et al., 2014; Piper et al., 2014).

To date, only very limited research work has been undertaken in the field of fNIRS-based deception decoding (Tian et al., 2009; Hu et al., 2012; Ding et al., 2013, 2014; Sai et al., 2014). In fact, no study has yet utilized or compared the fNIRS signals with physiological changes of a human body in a deception-decodingexperimental setting. There are only a few studies that have used physiological signals in parallel with fNIRS measurement (Falk et al., 2011; Zimmermann et al., 2013), and most of those were conducted only for BCI purposes.

In the present study, we performed offline single-trial classification of the truthful and deceptive responses of healthy male subjects. More specifically, we addressed the issues of (i) how truthful and deceptive responses are classified based on the fNIRS data collected from the prefrontal cortex (PFC), and (ii) how the inclusion of body physiological parameters including respiration, electrodermal activity (EDA) and body movement can affect classification accuracy. Linear discriminant analysis (LDA) was used to distinguish truthful from deceptive responses based on the fNIRS-determined mean and slope values of $\mathrm{HbO}$ combined with polygraph-based respiration and EDA results. The results obtained in this study demonstrate that the classification accuracy attained by the combined fNIRSpolygraph system was superior to that of either single system. To the best of our knowledge, this is the first time that polygraph has been combined with the fNIRS system in the lie-detection context.

\section{Materials and Methods}

\section{Subjects}

The experimentation was performed with 16 healthy male subjects (mean age: $31.2 \pm 3.2$ ). All had normal or corrected-tonormal vision. Among these 16 subjects, 13 were right handed and 3 were left handed. None of the subjects had a history of any neurological or psychiatric disorder. The Institutional Review Board of Pusan National University had approved this work. The subjects' written consent was obtained before the experiment, and the ethical standards of the latest Declaration of Helsinki were observed during the experiment.

\section{Experimental Procedure}

The experimental paradigm in this study is a modified version of the mock theft scenario presented by Tian et al. (2009), according to which the subject, left alone in a room, was instructed to steal either a $5000 \mathrm{KRW}$ note or a 10,000 KRW note from the drawer of a table and keep it in his pocket. The subject was then brought out of the room and taken to another room for questioning. The interrogator was totally unaware of the stolen note. Prior to the start of the formal deception experiment, each subject was given a brief description of the experiment, and a practice session was performed to confirm his complete understanding of the experimental procedure. The deception experiment required the subject to answer three types of questions (see Appendix in Supplementary Materials): "True/Lie" questions related to the 
stolen note and the note left in the drawer, neutral questions were general questions, answers to which were objectively "yes" or "no," and control questions relevant to the subject's personal life and some minor transgressive acts. The subject was instructed clearly that he has to deny the possession of the stolen note and had to answer a lie for only the questions related to the stolen note. In this study we only discriminate between the lie and the truth response of the subjects. The neutral and control questions were asked just to keep the subject active. A total of 10 question sessions, each session comprising five random questions, were presented on a computer screen. In each session, one true, one lie, one neutral, and two control questions were posed. Each question was displayed for about $3 \mathrm{~s}$, and the subject had to answer mentally as soon as he finished the reading. After each answer, a $15 \mathrm{~s}$ wait period was given for the settling of hemodynamic signals. There were two rest periods, each of $20 \mathrm{~s}$, given to the subject: one in the start of the experiment and the other one at the end of the experiment. In this study, six of the subjects took the $5000 \mathrm{KRW}$ note, and the other 10 took the 10,000 KRW note. Figure 1 illustrates the experimental procedure and a sample experimental trial.

\section{Data Acquisition}

A multi-channel continuous-wave imaging system developed by the authors (Bhutta et al., 2014) was used to acquire the brain signals. The optical probe of the fNIRS system was positioned on the forehead of the subject such that the emitters of the probe were in parallel to the FP1 and FP2 locations of the international 10-20 system. The hair was brushed backward to clear the forehead for attaching the flexible probe. The emitters and detectors were arranged to have good contact with the scalp and were then fixed to the subject's head with self-adhesive bandages. The probe configuration includes three near-infrared light-emitting diodes (LEDs) as emitters (each of which can emit three wavelengths: 640, 700, and $910 \mathrm{~nm}$ ) and eight Si-photodiodes as detectors, see Figure 2. The emitters and detectors were systematically positioned within a $5 \times 14 \mathrm{~cm}^{2}$ area according to the sourceto-detector distance of $3 \mathrm{~cm}$. Each LED was turned on and off sequentially, and the light diffused through the cortical region was detected at the nearest detectors. A total of 36 lightintensity signals ( 3 LEDs $\times 3$ wavelengths $\times 4$ neighboring detectors) were acquired at a sampling rate of $3.8 \mathrm{~Hz}$. A Velcro band kept the probe in the required position during the experiment.

To measure the physiological conditions of a human body (i.e., respiration, body movement, and skin conductance), we used the Paragon Acquisition System (PAS) developed by Limestone Technologies (Canada). The transducers were positioned as per the guidelines posted at the Limestone Technology website ${ }^{1}$. A Piezo Electronic StingRay_SE CM seat sensor, positioned on the chair where the subject would naturally rest most of his weight, recorded the subject's body movement. For two pneumatic respiration transducers provided in the PAS, one was affixed beneath the last rib and another one

${ }^{1}$ Limestone technologies website. http://www.limestonetech.com/index.cfm/ home/ near the top rib of the subject. Two EDA $24 \mathrm{~K}$ gold plated metal electrodes were used for maximal sensitivity to monitor the skin conductance or EDA. These electrodes were attached with a Velcro ring to the last two fingers of the subject's right hand. Micro-volt voltage was continuously applied between the electrodes, and the inter-electrode current was measured to acquire the EDA signal. All of these transducers were connected to the PAS via metal connectors, and the PAS was connected to the host PC through a USB cable. Figure 2 provides a complete block diagram of the system indicating not only the sensor positions but also the connections between the sensors and the host system.

\section{Data Processing}

The signals from both measuring systems (fNIRS, polygraph) were imported and further analyzed offline using MATLAB 7.9.0 (MathWorks, USA). The data were stored in a host-computer text file in the form of digitized raw intensity values from the fNIRS system. From these raw intensity values, the changes in optical density, $\triangle O D$, could be calculated at each discrete time $k$ as

$$
\Delta O D(k ; \lambda)=\log _{10} \frac{I_{0}(\lambda)}{I(k ; \lambda)}=l d(\lambda) \Delta \mu_{a}(k ; \lambda)
$$

where $I$ is the intensity of the detected light, $I_{0}$ is the intensity of the incident light, $d$ is the differential pathlength factor (DPF), $l$ is the distance between the emitter and the detector, and $\Delta \mu_{a}$ is the absorption change of the tissue. The changes of oxy-hemoglobin $\left(\Delta c_{H b O}\right)$ and deoxy-hemoglobin $\left(\Delta c_{H b R}\right)$ were measured using the modified Beer-Lambert law (Kocsis et al., 2006) as

$$
\begin{aligned}
{\left[\begin{array}{c}
\Delta c_{\mathrm{HbO}}(k) \\
\Delta c_{\mathrm{HbR}}(k)
\end{array}\right] } & =\left[\begin{array}{ll}
l d^{\lambda_{1}} \alpha_{\mathrm{HbO}}^{\lambda_{1}} & l d^{\lambda_{1}} \alpha_{\mathrm{HbR}}^{\lambda_{1}} \\
l d^{\lambda_{2}} \alpha_{\mathrm{HbO}}^{\lambda_{2}} & l d^{\lambda_{2}} \alpha_{\mathrm{HbR}}^{\lambda_{2}}
\end{array}\right]^{-1} \\
& \times\left[\begin{array}{c}
\Delta O D_{1}(k, \lambda) \\
\Delta O D_{2}(k, \lambda)
\end{array}\right]
\end{aligned}
$$

with $\lambda_{1}=640 \mathrm{~nm}, \lambda_{2}=910 \mathrm{~nm}, d^{\lambda 1}=6.63$, and $d^{\lambda 2}=2.765$ (Scholkmann and Wolf, 2013), according to the values for the wavelength-dependent absorption coefficients $\alpha_{H b O}, \alpha_{H b R}$ taken from the website of the University College London (Department of Medical Physics and Bioengineering, the title of the website: Specific Extinction Spectra of Tissue Chromophores) $)^{2}$. fNIRS, in detecting the hemodynamic response, picks up the physiological noises of respiration, pulse and low-frequency Mayer waves. To remove such noises, a second-order low-pass filter with a cutoff frequency of $0.15 \mathrm{~Hz}$ was used. Figure 3A shows the plots of averaged $\mathrm{HbO}$ signals for "true" and "lie" responses with its standard deviation (SD) and Figure 3B shows the averaged slope of the $\mathrm{HbO}$ signal for $2 \sim 7 \mathrm{~s}$ window out of the $15 \mathrm{~s}$ wait period with its SD. The data were averaged over all 10 trials and 16 subjects.

The PAS data were stored in a text file in the form of digitized values from the corresponding transducers. For simplicity and

\footnotetext{
${ }^{2}$ UCL Department of Medical Physics and Bioengineering, "Specific extinction spectra of tissue chromophores." http://www.medphys.ucl.ac.uk/research/borl/ research/NIR_topics/spectra/spectra.htm/
} 


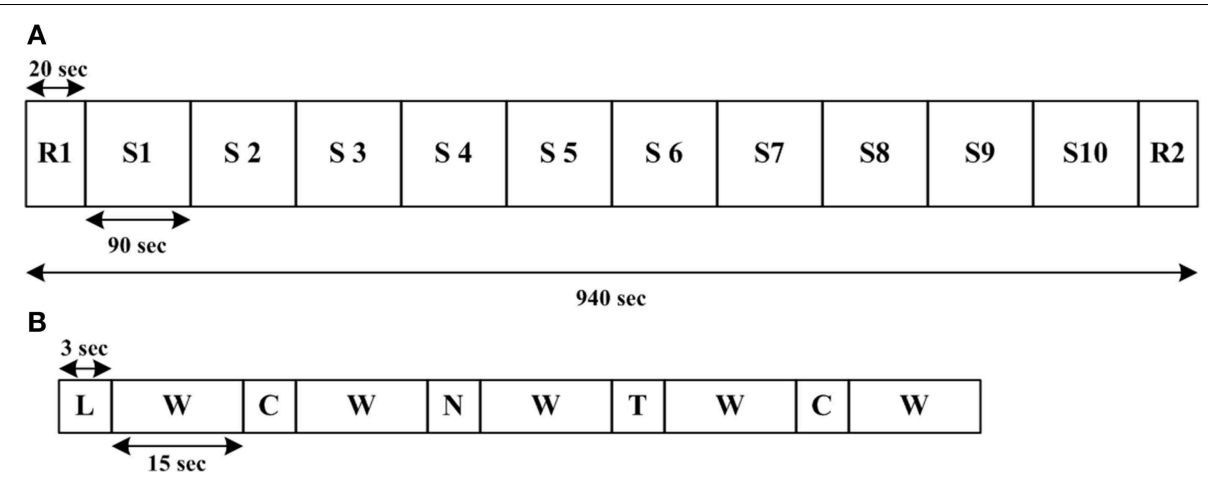

FIGURE 1 | Experimental procedure. (A) Total 10 sessions with two rest sessions: R for rest and $S$ for session, (B) a sample session: $L$ for lie, W for wait, $C$ for control, $\mathrm{N}$ for neutral, and $\mathrm{T}$ for true.

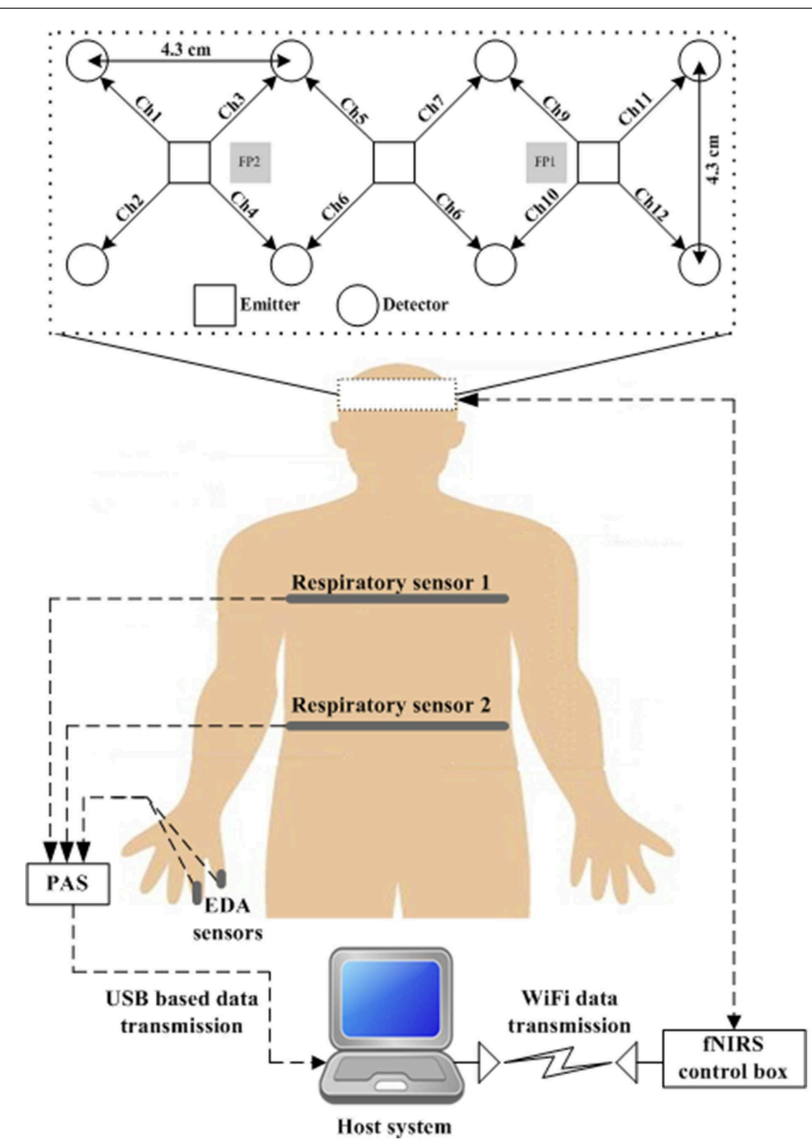

FIGURE 2 | Block diagram of measurement setup. The physiological signals (respiration and EDA) are transmitted to the Paragon Acquisition System (PAS), in which they are converted to digital data and then sent to the host system through a USB cable. The fNIRS probe is connected to the control box, which converts the analog light-intensity signals to digital signals and then transmits them to the host system by WiFi wireless transmission.

uniformity, the saved data were then normalized to a baseline for all of the transducers. Figure 4 depicts the averaged data for "true" and "lie" physiological responses with their SD, (Figure 4A: respiration amplitude, Figure 4B: EDA amplitude), recorded by the polygraph. The data were averaged over all 10 trials and 16 subjects.

\section{Classification}

In this study, LDA was used to distinguish "lie" from "true" responses because of its simplicity and execution speed. LDA is a linear classifier that discriminates between the different classes of data based on the hyper-planes (Lotte et al., 2007). The separating hyper-plane is designed in such a way that it should minimize the interclass variance and maximize the distance between the class means.

Classification was performed on the data segments acquired during the wait period after each true and lie response. For classification purpose we used 20 points (10 true and 10 lie) for each subject, as shown in Figure 1, there are 10 session of our experiment and each session has at least one true and one lie question in it. The classification accuracies were calculated using 10 runs of a ten-fold cross-validation, that is, one truth/lie trial was selected for the testing purpose whereas the classifier was trained on the remaining nine trials. This process was repeated for all the trials. The classification accuracies were determined with the error averaged over all training/testing combinations.

In this study, only $\mathrm{HbO}$ signals were considered for classification purposes of fNIRS data. Signal mean (SM) and signal slope (SS) of $\mathrm{HbO}$ are used as classification features. A grand average of $\mathrm{HbO}$ was taken over all 12 channels. The SS value for each wait period was determined by fitting a line to all the data points during the $2 \sim 7 \mathrm{~s}$ window of the wait period, using the linear regression. Hong et al. (2015) has shown that SM and SS give the best result when a window of $2 \sim 7 \mathrm{~s}$ after the stimulus is used for getting SM and SS. The SM value for each wait period was determined by averaging the data points in the respective time windows.

In this study, respiration and EDA signals were used for classification of polygraph data. The motion sensor values were excluded from the classification process owing to the lack of significant difference between the recorded "true" and "lie" response readings. For the polygraph data, only the first $7 \mathrm{~s}$ of each wait period after the true and lie questions were analyzed. As two respiratory sensors were used, the average value over 


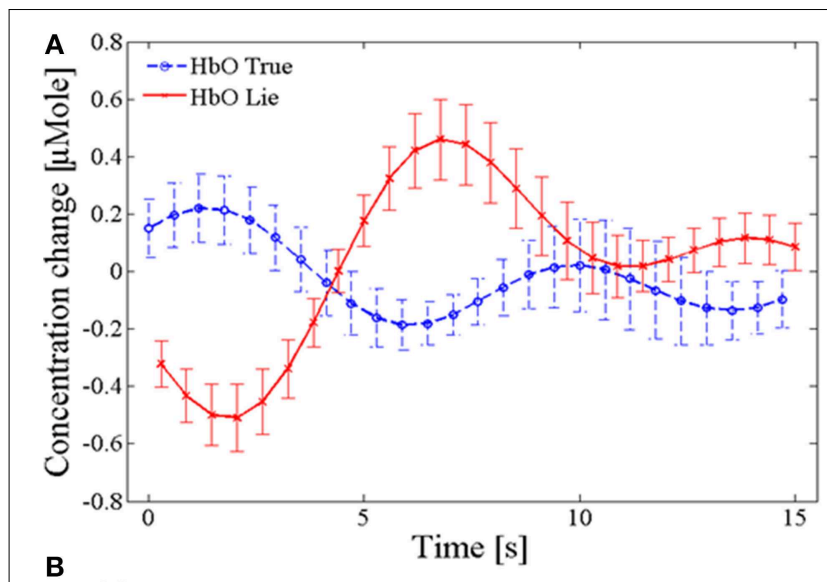

.

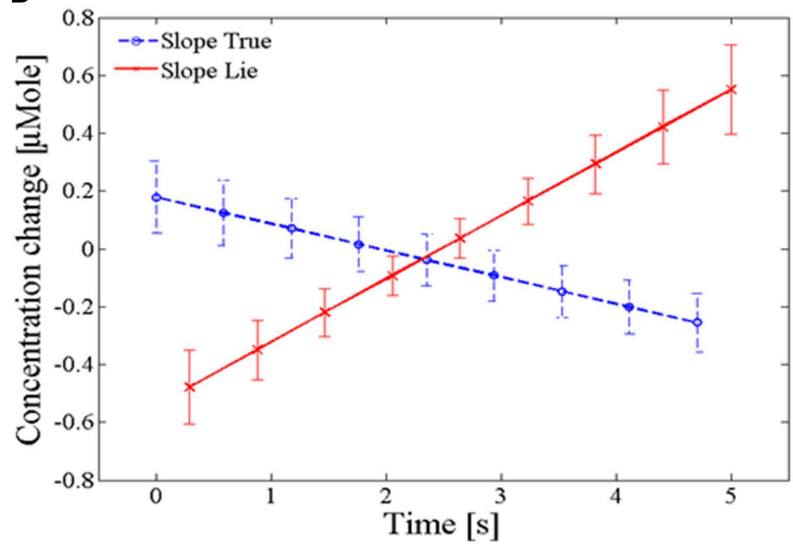

FIGURE 3 | Averaged oxy-hemoglobin responses. (A) Comparison of $\mathrm{HbO}$ means for true and lie responses during the $15 \mathrm{~s}$ wait period in Figure 1B, (B) comparison of $\mathrm{HbO}$ slopes during the early $2 \sim 7 \mathrm{~s}$ interval out of the $15 \mathrm{~s}$ wait period. Data were averaged over all 10 trials and 16 subjects (i.e., total 160 responses for each lie and true).

both sensors was taken. The mean values of respiration and EDA signals were used as classifier features for the polygraph data. The mean values were calculated by averaging the data points during the $7 \mathrm{~s}$ time window.

After calculating the classification results for individual modalities (fNIRS, polygraph), a combined classification accuracy was also calculated by using LDA as meta-classifier. Then, the features used in individual calculations were combined to get the combined classification accuracy.

\section{Results}

Figure 5 shows the two classes (true and lie) obtained after classification of the $\mathrm{HbO}$ mean and $\mathrm{HbO}$ slope data for the true and lie data of Subject 15. Figure 6 shows the same two classes after classification of the same subject's respiration and EDA data values. It can be seen from Figures 5, 6 that the feature values were scaled between 0 and 1 using the equation below.

$$
s^{\prime}=\frac{s-\min (s)}{\max (s)-\min (s)}
$$

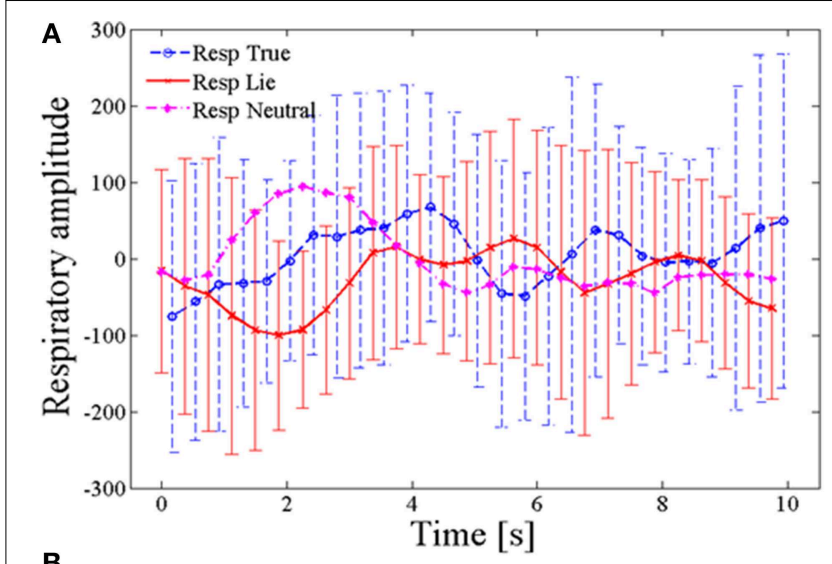

B

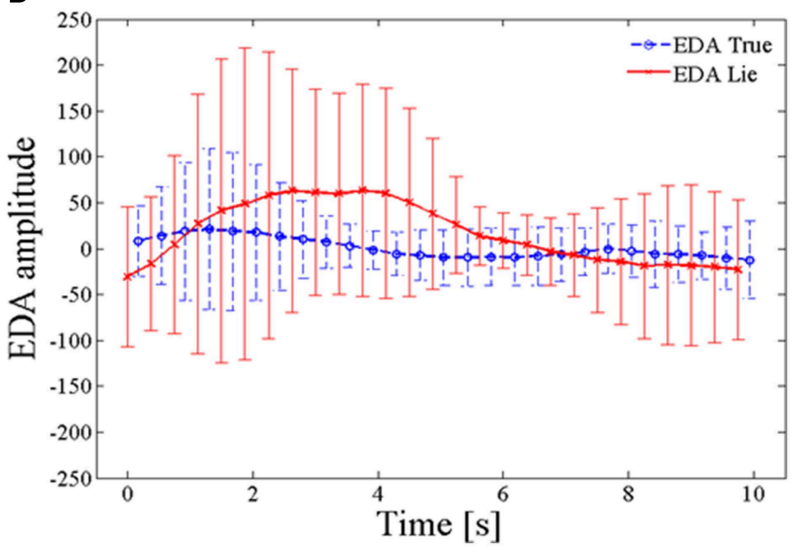

FIGURE 4 | Averaged polygraph-based physiological responses. (A) Averaged respiratory responses for lie, true, and neutral questions, (B) averaged EDA responses for lie and true. Data were averaged over all 10 trials and 16 subjects.

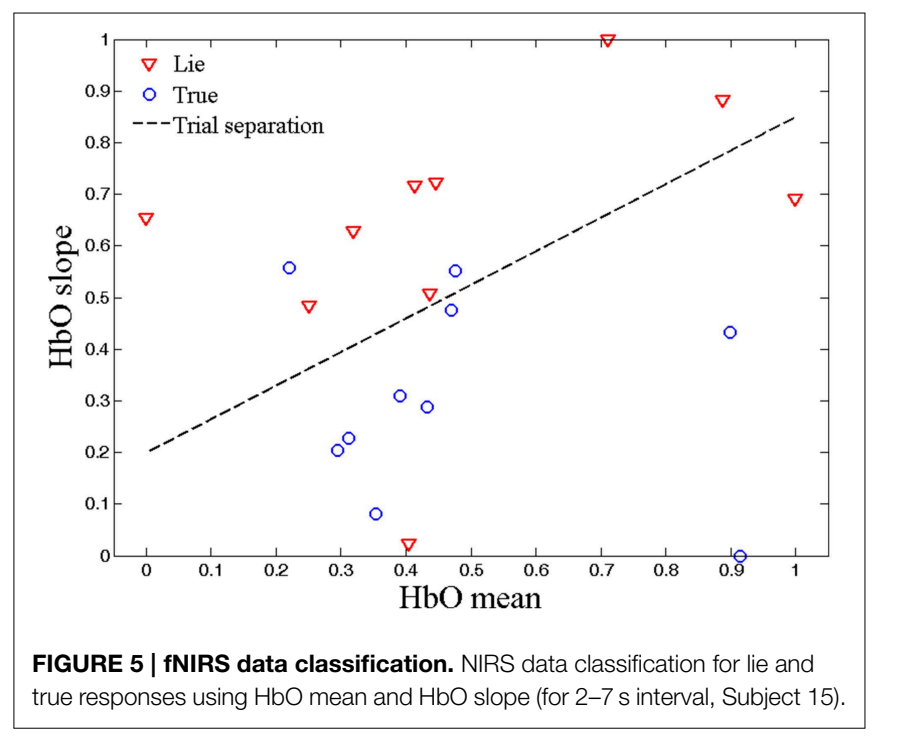

where $s$ denotes the original data for each feature, $s^{\prime}$ denotes the scaled value of the respective feature, $\min (s)$ and $\max (s)$ denote the minimum and maximum values in the respective feature set. 
To compare the results obtained by the combined system and individual systems, the true and lie responses were classified once again using four features ( $\mathrm{HbO}$ mean, $\mathrm{HbO}$ slope, respiration, and EDA mean values): Figure 7 compares the classification accuracies of individual systems and the combined system. As averaged over all 16 subjects, the classification accuracies obtained from individual fNIRS and polygraph modalities were 71.6 and $74.5 \%$, respectively, whereas with the combined fNIRSpolygraph system, a very considerable improvement, to $86.5 \%$, was achieved. The classification accuracies obtained by the combined fNIRS-polygraph system were compared with those obtained by the individual systems using the non-parametric Mann-Whitney $U$-test. The results for the combined system vs.

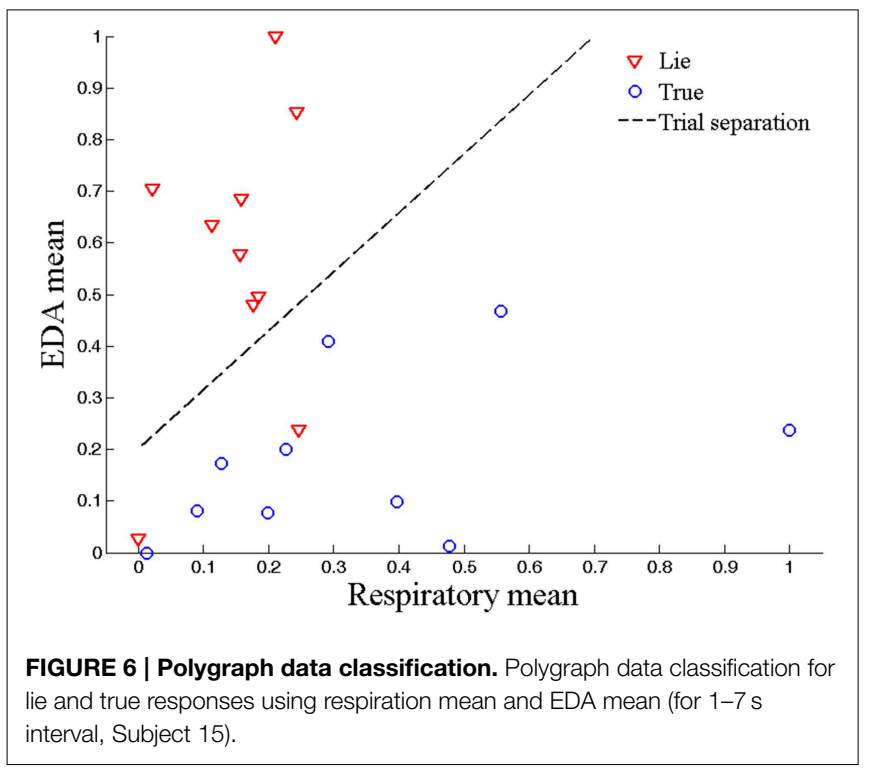

fNIRS alone were $U_{(16)}=256$ and $p<0.0001$, whereas those for the combined system vs. the polygraph alone were $U_{(16)}=252.5$ and $p=0.0001$.

\section{Discussion}

The polygraph is widely used in interrogations (i.e., criminal investigations) and office-interview settings to detect lies (Ginton, 2013). The major physiological responses measured are respiration, heart rate, skin conductance, body movement, and blood pressure. In this study, a commercially available polygraph machine, the Paragon Acquisition System (PAS), was used to detect the physiological signals. Respiration, electrodermal activity, and body movement were measured from the respective measuring points shown in Figure 2. The body movement data were excluded from the analysis, owing to the lack of difference in detected data. The reason for this might have been the fact that for the fNIRS measurements, the subjects were asked to remain still and not move their body, as this can generate noise in fNIRS data. The measured signals from the respiration and EDA transducers were fed to the PSA, which converted them to digital and then sent them to the host system using a wired connection.

Figure 4 shows a clear and significant difference between the respiration and EDA signals corresponding to true and lie responses. It can be seen from the plot that in the first few seconds of the wait period the respiration amplitude for lie is relatively lower than the amplitude during the truth and the EDA signals goes high during the first few seconds of the lie condition. These findings are in accordance with the previous literature (Matsuda et al., 2011). These are only some of the physiological measures for lie detection; nevertheless, scientists believe that provided the brain signals activated during the deception process can be clearly observed, understanding the deception phenomenon and detecting them are easily done. In the present study, for

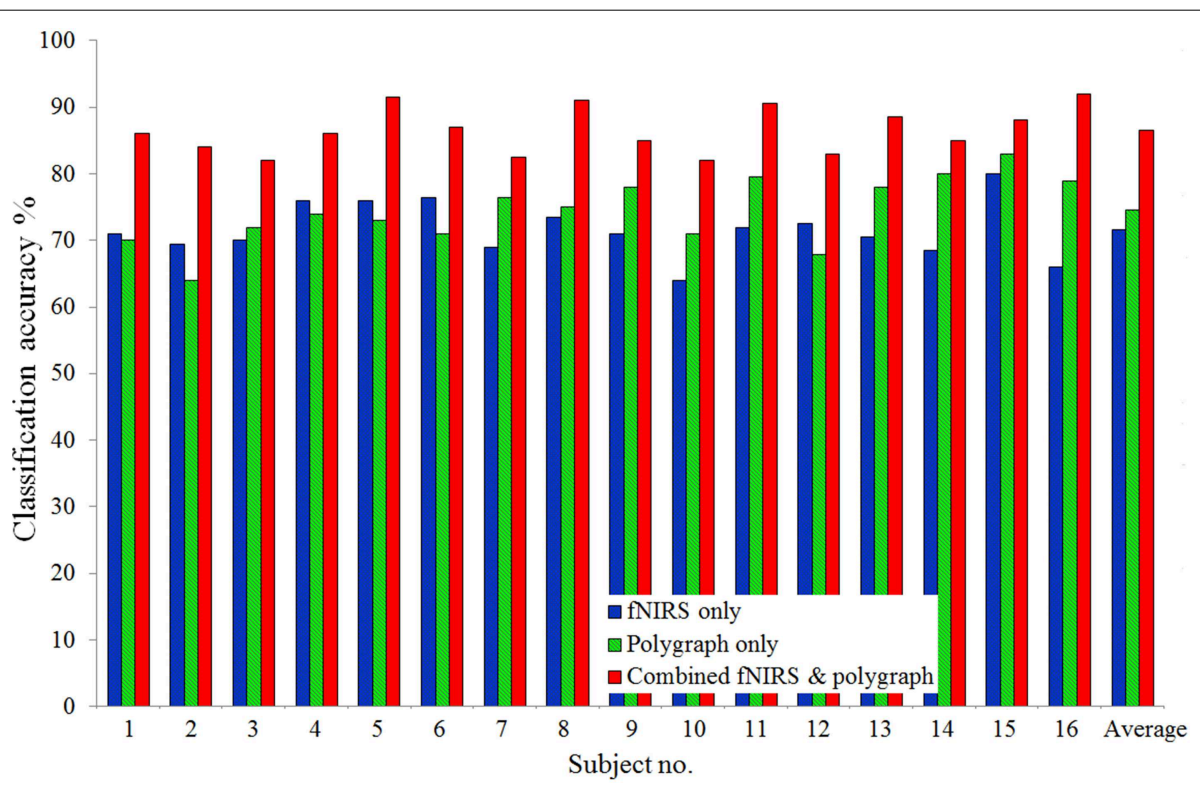

FIGURE 7 | Comparison. Comparison of classification accuracies of individual modalities and the combined system. 
direct detection of brain signals, the fNIRS system was used to acquire hemodynamic signals from the brain's PFC. There are multiple areas in the brain that are involved in lying but the previous studies have already established that the PFC is activated when an individual practices deception (Christ et al., 2009; Hu et al., 2012; Ding et al., 2014; Farah et al., 2014; Guhn et al., 2014; Sai et al., 2014). Figure 3 shows the clear and significant difference between the $\mathrm{HbO}$ signals corresponding to lie and true responses. It serves to demonstrate that true and lie responses can be separated from each other based on the respective relevant hemodynamic responses from the PFC.

The classification was performed using LDA to distinguish HbO signals (as fNIRS data features) along with respiration and EDA signals (as polygraph data features). Figures 5, 6 show the two different classifications (true and lie) obtained after classification of the fNIRS data and polygraph data, respectively, for the same subject (Subject 15). The separability of the hemodynamic and physiological responses arising during the lie and true responses is clear.

This is the first study in which fNIRS and polygraph are combined to detect deception. As noted above, fNIRS detects the brain activity occurring during the deception process, while polygraph detects the physiological response from the body. It was assumed that by combining the two methodologies, system performance would be improved and, in fact, the data collected during this study proved the correctness of the assumption. Figure 7 compares the classification accuracies of the single measuring systems and that of the combined fNIRS-polygraph system. The averaged classification accuracies of individual fNIRS and polygraph were 71.6 and $74.5 \%$, respectively; but that of the combined fNIRS-polygraph system, remarkably, was $86.5 \%$. It was observed that, in some trials, fNIRS was unable to detect the deception but PAS was able to detect it and vice versa. These results prove emphatically that the combined system is more efficient in discriminating between true and lie responses.

As polygraph is widely used in criminal investigations and court settings, the observed $74.5 \%$ classification accuracy of polygraph seems rather weak. The reason for this low accuracy can be because, the used questions from Tian et al. (2009) in this paper were not so challenging. Probably, in the criminal investigations, the questions are well arranged in relation to the crime details involved. Recently, Meijer et al. (2014) published a paper, in which the authors have conducted a meta-analysis for the verification of different deception decoding paradigms using four physiological measures: skin conductance, respiratory response, heart rate, and P300 component of EEG. They have conducted their analysis over more than hundred published studies from $1970 \sim 2013$. The average correct detection rate for guilty and innocent subjects using skin conductance and respiratory response ranges from 80 to $85 \%$ for all studies. The $74.5 \%$ accuracy found in our study is comparable to the average obtained by the above mentioned study. An important point should also be noted that, the results of this study cannot be directly compared with the results of previous studies because the classification accuracies reported in this study refer to differentiation between responses within individuals, whereas most previous studies deal with differentiating between deceptive and truthful individuals or groups.

All of the data analysis was performed offline, due to the limitation of the PAS, which provides data only after completion of an experiment. This limitation will be overcome by using a system that can provide physiological measurements in real time. Also, the present experiments were performed only with male subjects aged $27-34$, though differences in the hemodynamic responses of females and older individuals relative to those of young males has already been reported (Anokhin et al., 1996; May et al., 2014; Zhou et al., 2014). One additional factor that should be noted is that all of our subjects were university graduate students with no criminal background. Considering that lie-detection systems are used mostly in investigations of criminals, the great majority of whom are not well educated and, often, inured to the criminal-investigative process, further research will have to be done to test the performance of our proposed combined fNIRS-polygraph system for real criminalinvestigative contexts.

\section{Conclusions}

In this study, a combined fNIRS-polygraph system for singletrial lie detection was compared with each fNIRS and polygraph system. The fNIRS system decodes deception based on the hemodynamic changes of oxy-hemoglobin measured at the prefrontal cortex, and the polygraph operates on the basis of physiological responses from the body such as respiration and electrodermal activities. Both modalities were timesynchronized, and the data were classified first separately for each system and then combined using the linear discriminant analysis as a classifier. The classification accuracy achieved by the combined system was much higher than those achieved by the single systems. These results indicate that the combined fNIRS-polygraph system offers a great potential for application to real-life lie-detection contexts.

\section{Author Contributions}

MB performed the experiment and carried out the data processing, $\mathrm{MH}$ and $\mathrm{YK}$ reviewed the manuscript and suggested a number of critical methodologies to improve the manuscript, and $\mathrm{KH}$ supervised the research and corrected the entire manuscript. All of the authors read and approved the final manuscript.

\section{Acknowledgments}

This work was supported by the National Research Foundation of Korea under the Ministry of Science, ICT and Future Planning, Korea (grant no. NRF-2014-R1A2A1A10049727 and NRF-2014R1A2A1A01005128).

\section{Supplementary Material}

The Supplementary Material for this article can be found online at: http://journal.frontiersin.org/article/10.3389/fpsyg. 2015.00709/abstract 


\section{References}

Anokhin, A. P., Birbaumer, N., Lutzenberger, W., Nikolaev, A., and Vogel, F. (1996). Age increases brain complexity. Electroen. Clin. Neuro. 99, 63-68. doi: 10.1016/0921-884X(96)95573-3

Barbour, R. L., Graber, H. L., Pei, Y., Zhong, S., and Schmitz, C. H. (2001). Optical tomographic imaging of dynamic features of dense-scattering media. J. Opt. Soc. Am. A. 18, 3018-3036. doi: 10.1364/JOSAA. 18.003018

Bhutta, M. R., Hong, K.-S., Kim, B.-M., Hong, M. J., Kim, Y.-H., and Lee, S.H. (2014). Note: Three wavelengths near-infrared spectroscopy system for compensating the light absorbance by water. Rev. Sci. Instrum. 85:026111. doi: $10.1063 / 1.4865124$

Cantilena, L., Kahn, R., Duncan, C. C., Li, S. H., Anderson, A., and Elkashef, A. (2012). Safety of atomoxetine in combination with intravenous cocaine in cocaine-experienced participants. J. Addict. Med. 6, 265-273. doi: 10.1097/ADM.0b013e31826b767f

Christ, S. E., Van Essen, D. C., Watson, J. M., Brubaker, L. E., and McDermott, K. B. (2009). The contributions of prefrontal cortex and executive control to deception: evidence from activation likelihood estimate meta-analyses. Cereb. Cortex 19, 1557-1566. doi: 10.1093/cercor/bhn189

Ding, X. P., Gao, X., Fu, G., and Lee, K. (2013). Neural correlates of spontaneous deception: a functional near-infrared spectroscopy (fNIRS) study. Neuropsychologia 51, 704-712. doi: 10.1016/j.neuropsychologia.2012.12.018

Ding, X. P., Sai, L., Fu, G., Liu, J., and Lee, K. (2014). Neural correlates of secondorder verbal deception: a functional near-infrared spectroscopy (fNIRS) study. Neuroimage 87, 505-514. doi: 10.1016/j.neuroimage.2013.10.023

Duncan, C. C., Barry, R. J., Connolly, J. F., Fischer, C., Michie, P. T., Naatanen, R., et al. (2009). Event-related potentials in clinical research: guidelines for eliciting, recording, and quantifying mismatch negativity, P300, and N400. Clin. Neurophysiol. 120, 1883-1908. doi: 10.1016/j.clinph.2009.07.045

Falk, T. H., Guirgis, M., Power, S., and Chau, T. T. (2011). Taking NIRSBCIs outside the lab: towards achieving robustness against environment noise. IEEE Trans. Neural Syst. Rehabil. Eng. 19, 136-146. doi: 10.1109/TNSRE. 2010.2078516

Farah, M. J., Hutchinson, J. B., Phelps, E. A., and Wagner, A. D. (2014). Functional MRI-based lie detection: scientific and societal challenges. Nat. Rev. Neurosci. 15, 123-131. doi: $10.1038 / \mathrm{nrn} 3665$

Farahani, E. D., and Moradi, M. H. (2013). A concealed information test with combination of ERP recording and autonomic measurements. Neurophysiology 45, 223-233. doi: 10.1007/s11062-013-9360-y

Farwell, L. A., and Donchin, E. (1991). The truth will out: interrogative polygraphy ("lie detection") with event-related brain potentials. Psychophysiology 28, 531-547. doi: 10.1111/j.1469-8986.1991.tb01990.x

Fazli, S., Mehnert, J., Steinbrink, J., Curio, G., Villringer, A., Muller, K. R., et al. (2012). Enhanced performance by a hybrid NIRS-EEG brain computer interface. Neuroimage 59, 519-529. doi: 10.1016/j.neuroimage.2011.07.084

Ginton, A. (2013). A non-standard method for estimating accuracy of lie detection techniques demonstrated on a self-validating set of field polygraph examinations. Psychol. Crime Law 19, 577-594. doi: 10.1080/1068316X.2012.656118

Guhn, A., Dresler, T., Andreatta, M., and Muller, L. D. (2014). Medial prefrontal cortex stimulation modulates the processing of conditioned fear. Front. Behav. Neurosci. 8:44. doi: 10.3389/fnbeh.2014.00044

Hong, K.-S., and Nguyen, H. D. (2014). State-space models of impulse hemodynamic responses over motor, somatosensory, and visual cortices. Biomed. Opt. Express 5, 1778-1798. doi: 10.1364/BOE.5.001778

Hong, K.-S., Naseer, N., and Kim, Y.-H. (2015). Classification of prefrontal and motor cortex signals for three-class fNIRS-BCI. Neurosci. Lett. 587, 87-92. doi: 10.1016/j.neulet.2014.12.029

Hu, X. S., Hong, K.-S., and Ge, S. S. (2011). Recognition of stimulusevoked neuronal optical response by identifying chaos levels of nearinfrared spectroscopy time series. Neurosci. Lett. 504, 115-120. doi: 10.1016/j.neulet.2011.09.011

Hu, X. S., Hong, K.-S., and Ge, S. S. (2012). fNIRS-based online deception decoding. J. Neural Eng. 9:026012. doi: 10.1088/1741-2560/9/2/026012

Irani, F., Platek, S. M., Bunce, S., Ruocco, A. C., and Chute, D. (2007). Functional near infrared spectroscopy (fNIRS): an emerging neuroimaging technology with important applications for the study of brain disorders. Clin. Neuropsychol. 21, 9-37. doi: 10.1080/13854040600910018

Jung, C. G. (1919). Studies in Word-Association: Experiments in the Diagnosis of Psychopathological Conditions Carried Out at the Psychiatric Clinic of the University of Zurich, Under the Direction of C. G. Jung... Authorized Translation by Dr. M. D. Eder. New York, NY: Moffat, Yard and Company.

Kamran, M. A., and Hong, K.-S. (2013). Linear parameter-varying model and adaptive filtering technique for detecting neuronal activities: an fNIRS study. J. Neural Eng. 10:056002. doi: 10.1088/1741-2560/10/5/056002

Khan, M. J., Hong, M. J., and Hong, K.-S. (2014). Decoding of four movement directions using hybrid NIRS-EEG brain-computer interface. Front. Hum. Neurosci. 8:244. doi: 10.3389/fnhum.2014.00244

Kim, C.-K., Lee, S., Koh, D., and Kim, B.-M. (2011). Development of wireless NIRS system with dynamic removal of motion artifacts. Biomed. Eng. Lett. 1, 254-259. doi: 10.1007/s13534-011-0042-7

Kochel, A., Plichta, M. M., Schafer, A., Leutgeb, V., Scharmuller, W., Fallgatter, A. J., et al. (2011). Affective perception and imagery: a NIRS study. Int. J. Psychophysiol. 80, 192-197. doi: 10.1016/j.ijpsycho.2011.03.006

Kocsis, L., Herman, P., and Eke, A. (2006). The modified Beer-Lambert law revisited. Phys. Med. Biol. 51, N91-N98. doi: 10.1088/0031-9155/51/5/N02

Lanfranconi, F., Pollastri, L., Ferri, A., Fraschini, D., Masera, G., and Miserocchi, G. (2014). Near infrared spectroscopy (NIRS) as a new noninvasive tool to detect oxidative skeletal muscle impairment in children survived to acute lymphoblastic leukaemia. PLOS ONE 9:99282. doi: 10.1371/journal.pone.0099282

Liang, P., Jia, X., Taatgen, N. A., Zhong, N., and Li, K. (2014). Different strategies in solving series completion inductive reasoning problems: an fMRI and computational study. Int. J. Psychophysiol. 93, 253-260. doi: 10.1016/j.ijpsycho.2014.05.006

Lotte, F., Congedo, M., Lecuyer, A., Lamarche, F., and Arnaldi, B. (2007). A review of classification algorithms for EEG-based brain-computer interfaces. J. Neural Eng. 4, R1-R13. doi: 10.1088/1741-2560/4/R01

Matsuda, I., Nittono, H., and Ogawa, T. (2011). Event-related potentials increase the discrimination performance of the autonomic-based concealed information test. Psychophysiology 48, 1701-1710. doi: 10.1111/j.14698986.2011.01266.x

Matsuda, I., Nittono, H., and Ogawa, T. (2013). Identifying concealment-related responses in the concealed information test. Psychophysiology 50, 617-626. doi: 10.1111/psyp. 12046

May, A. C., Stewart, J. L., Tapert, S. E., and Paulus, M. P. (2014). The effect of age on neural processing of pleasant soft touch stimuli. Front. Behav. Neurosci. 8:52. doi: $10.3389 /$ fnbeh.2014.00052

Meijer, E. H., Selle, N. K., Elber, L., and Ben-Shakhar, G. (2014). Memory detection with the Concealed Information Test: a meta analysis of skin conductance, respiration, heart rate, and P300 data. Psychophysiology 51, 879-904. doi: 10.1111/psyp.12239

Mohamed, F. B., Faro, S. H., Gordon, N. J., Platek, S. M., Ahmad, H., and Williams, J. M. (2006). Brain mapping of deception and truth telling about an ecologically valid situation: functional $\mathrm{MR}$ imaging and polygraph investigationinitial experience. Radiology 238, 679-688. doi: 10.1148/radiol.2382 050237

Muehlemann, T., Haensse, D., and Wolf, M. (2008), Wireless miniaturized in-vivo near infrared imaging. Opt. Express 16, 10323-10330. doi: 10.1364/OE.16.010323

Naseer, N., and Hong, K.-S. (2013). Classification of functional near-infrared spectroscopy signals corresponding to the right-and left-wrist motor imagery for development of a brain-computer interface. Neurosci. Lett. 553, 84-89. doi: 10.1016/j.neulet.2013.08.021

Naseer, N., and Hong, K.-S. (2015a). fNIRS-based brain-computer interfaces: a review. Front. Hum. Neurosci. 9:3. doi: 10.3389/fnhum.2015.00003

Naseer, N., Hong, M. J., and Hong, K.-S. (2014). Online binary decision decoding using functional near-infrared spectroscopy for the development of braincomputer interface. Exp. Brain Res. 232, 555-564. doi: 10.1007/s00221-0133764-1

Naseer, N., and Hong, K.-S. (2015b). Decoding answers to four-choice questions using functional near-infrared spectroscopy. J. Near Infrared Spectrosc. 23, 23-31. doi: 10.1255/jnirs.1145 
Piper, S. K., Krueger, A., Koch, S. P., Mehnert, J., Habermehl, C., Steinbrink, J., et al. (2014). A wearable multi-channel fNIRS system for brain imaging in freely moving subjects. Neuroimage 85, 64-71. doi: 10.1016/j.neuroimage.2013.06.062

Quaresima, V., Lepanto, R., and Ferrari, M. (2003). The use of near infrared spectroscopy in sports medicine. J. Sports Med. Phys. Fit. 43, 1-13.

Roos, A., Robertson, F., Lochner, C., Vythilingum, B., and Stein, D. J. (2011). Altered prefrontal cortical function during processing of fear-relevant stimuli in pregnancy. Behav. Brain Res. 222, 200-205. doi: 10.1016/j.bbr.2011.03.055

Rosenfeld, J. P., Hu, X., and Pederson, K. (2012). Deception awareness improves P300-based deception detection in concealed information tests. Int. J. Psychophysiol. 86, 114-121. doi: 10.1016/j.ijpsycho.2012.06.007

Rosenfeld, J. P., Hu, X., Labkovsky, E., Meixner, J., and Winograd, M. R. (2013). Review of recent studies and issues regarding the P300-based complex trial protocol for detection of concealed information. Int. J. Psychophysiol. 90, 118-134. doi: 10.1016/j.ijpsycho.2013.08.012

Sai, L., Zhou, X., Ding, X. P., Fu, G., and Sang, B. (2014). Detecting concealed information using functional near-infrared spectroscopy. Brain Topogr. 27, 652-662. doi: 10.1007/s10548-014-0352-Z

Santosa, H., Hong, M. J., and Hong, K.-S. (2014). Lateralization of music processing with noises in the auditory cortex: an fNIRS study. Front. Behav. Neurosci. 8:418. doi: 10.3389/fnbeh.2014.00418

Santosa, H., Hong, M. J., Kim, S. P., and Hong, K.-S. (2013). Noise reduction in functional near-infrared spectroscopy signals by independent component analysis. Rev. Sci. Instrum. 84, 073106. doi: 10.1063/1.4812785

Scholkmann, F., and Wolf, M. (2013). General equation for the differential pathlength factor of the frontal human head depending on wavelength and age. J. Biomed. Opt. 18:105004. doi: 10.1117/1.JBO.18.10.105004

Soekadar, S. R., Witkowski, M., Cossio, E. G., Birbaumer, N., and Cohen, L. G. (2014). Learned EEG-based brain self-regulation of motor-related oscillations during application of transcranial electric brain stimulation: feasibility limitations. Front. Behav. Neurosci. 8:93. doi: 10.3389/fnbeh.2014.00093

Spence, S. A., Hunter, M. D., Farrow, T. F., Green, R. D., Leung, D. H., Hughes, C. J., et al. (2004). A cognitive neurobiological account of deception: evidence from functional neuroimaging. Philos. Trans. R. Soc. B Biol. Sci. 359, 1755-1762. doi: $10.1098 /$ rstb.2004.1555

Taillefer, M. C., and Denault, A. Y. (2005). Cerebral near-infrared spectroscopy in adult heart surgery: systematic review of its clinical efficacy. Can. J. Anesth. 52, 79-87. doi: 10.1007/BF03018586

Tempest, G. D., Eston, R. G., and Parfitt, G. (2014). Prefrontal cortex haemodynamics and affective responses during exercise: a multi-channel near infrared spectroscopy study. PLoS ONE 9:95924. doi: 10.1371/journal.pone.0095924

Tian, F., Sharma, V., Kozel, F. A., and Liu, H. (2009). Functional near-infrared spectroscopy to investigate hemodynamic responses to deception in the prefrontal cortex. Brain Res. 1303, 120-130. doi: 10.1016/j.brainres.2009. 09.085

Turnip, A., Hong, K.-S., and Jeong, M.-Y. (2011). Real-time feature extraction of P300 component using adaptive nonlinear principal component analysis. Biomed. Eng. Online 10:83. doi: 10.1186/1475-925X-10-83

Walczyk, J. J., Igou, F. P., Dixon, A. P., and Tcholakian, T. (2013). Advancing lie detection by inducing cognitive load on liars: a review of relevant theories and techniques guided by lessons from polygraph-based approaches. Front. Psychol. 4:14. doi: 10.3389/fpsyg.2013.00014

Wolf, M., Ferrari, M., and Quaresima, V. (2007). Progress of near-infrared spectroscopy and topography for brain and muscle clinical applications. J. Biomed. Opt. 12, 062104. doi: 10.1117/1.2804899

Yuan, Z., and Ye, J.-C. (2013). Fusion of fNIRS and fMRI data: identifying when and where hemodynamic signals are changing in human brains. Front. Hum. Neurosci. 7:676. doi: 10.3389/fnhum.2013.00676

Zhao, M., Zheng, C., and Zhao, C. (2012). A new approach for concealed information identification based on ERP assessment. J. Med. Syst. 36, 2401-2409. doi: 10.1007/s10916-011-9707-0

Zhou, Y., Li, S., Dunn, J., Li, H. D., Qin, W., Zhu, M. H., et al. (2014). The neural correlates of risk propensity in males and females using resting-state fMRI. Front. Behav. Neurosci. 8:2. doi: 10.3389/fnbeh.2014.00002

Zimmermann, R., Marchal-Crespo, L., Edelmann, J., Lambercy, O., Fluet, M. C., Riener, R., et al. (2013). Detection of motor execution using a hybrid fNIRSbiosignal BCI: a feasibility study. J. NeuroEng. Rehabil. 10:4. doi: 10.1186/17430003-10-4

Conflict of Interest Statement: The authors declare that the research was conducted in the absence of any commercial or financial relationships that could be construed as a potential conflict of interest.

Copyright (c) 2015 Bhutta, Hong, Kim and Hong. This is an open-access article distributed under the terms of the Creative Commons Attribution License (CC BY). The use, distribution or reproduction in other forums is permitted, provided the original author(s) or licensor are credited and that the original publication in this journal is cited, in accordance with accepted academic practice. No use, distribution or reproduction is permitted which does not comply with these terms. 